\title{
Can we infer plant facilitation from remote sensing? a test across global drylands
}

\author{
Chi Xu,${ }^{1,2,12}$ Milena Holmgren, ${ }^{3}$ Egbert H. Van Nes,${ }^{2}$ Fernando T. Maestre ${ }^{4}$ Santiago Soliveres, ${ }^{5}$ Miguel \\ Berdugo, ${ }^{4}$ Sonia Kéfi,${ }^{6}$ Pablo A. Marquet, ${ }^{7,8,9,10,11}$ Sebastián Abades,${ }^{7,8,9}$ and Marten Scheffer ${ }^{2}$ \\ ${ }^{1}$ School of Life Sciences, Nanjing University, 163 Xianlin Road, Nanjing 210023 People's Republic of China \\ ${ }^{2}$ Aquatic Ecology and Water Quality Management Group, Wageningen University, P.O. Box 47, NL-6700 AA, \\ Wageningen, The Netherlands \\ ${ }^{3}$ Resource Ecology Group, Wageningen University, P.O. Box 47, NL-6700 AA, Wageningen, The Netherlands \\ ${ }^{4}$ Área de Biodiversidad y Conservación, Departamento de Biología y Geología, Física y Química Inorgánica, \\ Universidad Rey Juan Carlos, c/Tulipán s/n., E-28933 Móstoles, Spain \\ ${ }^{5}$ Institute of Plant Sciences, University of Bern, Altenbergrain 21, 3013 Bern, Switzerland \\ ${ }^{6}$ Institut des Sciences de l'Evolution, Université de Montpellier, CNRS, IRD, EPHE, CCO65, \\ Place Eugène Bataillon, 34095 Montpellier Cedex 05, France \\ ${ }^{7}$ Departamento de Ecología, Facultad de Ciencias Biológicas, Pontificia Universidad Católica de Chile, Alameda 340, Santiago, Chile \\ ${ }^{8}$ Instituto de Ecología y Biodiversidad (IEB), Casilla 653, Santiago, Chile \\ ${ }^{9}$ Laboratorio Internacional en Cambio Global (LINCGlobal) Pontificia Universidad Católica de Chile, Alameda 340, Santiago, Chile \\ ${ }^{10}$ The Santa Fe Institute, 1399 Hyde Park Road, Santa Fe, New Mexico 87501 USA \\ ${ }^{11}$ Centro Cambio Global UC (PUC-Global) Vicuña Mackenna 4860, Macul, Santiago, Chile
}

\begin{abstract}
Facilitation is a major force shaping the structure and diversity of plant communities in terrestrial ecosystems. Detecting positive plant-plant interactions relies on the combination of field experimentation and the demonstration of spatial association between neighboring plants. This has often restricted the study of facilitation to particular sites, limiting the development of systematic assessments of facilitation over regional and global scales. Here we explore whether the frequency of plant spatial associations detected from high-resolution remotely sensed images can be used to infer plant facilitation at the community level in drylands around the globe. We correlated the information from remotely sensed images freely available through Google Earth with detailed field assessments, and used a simple individualbased model to generate patch-size distributions using different assumptions about the type and strength of plant-plant interactions. Most of the patterns found from the remotely sensed images were more right skewed than the patterns from the null model simulating a random distribution. This suggests that the plants in the studied drylands show stronger spatial clustering than expected by chance. We found that positive plant co-occurrence, as measured in the field, was significantly related to the skewness of vegetation patch-size distribution measured using Google Earth images. Our findings suggest that the relative frequency of facilitation may be inferred from spatial pattern signals measured from remotely sensed images, since facilitation often determines positive co-occurrence among neighboring plants. They pave the road for a systematic global assessment of the role of facilitation in terrestrial ecosystems.
\end{abstract}

Key words: arid ecosystems; community structure; competition; desertification; individual-based model; patch-size distribution; positive interactions; spatial pattern; vegetation pattern.

\section{INTRODUCTION}

The development of remote sensing tools and freely available resources on the internet has made it increasingly feasible to retrieve systematic information about vegetation patterning at fine spatial resolutions in terrestrial ecosystems worldwide (e.g., Thompson and Katul 2011, Cramer and Barger 2013). It has been suggested that such patterns might be used to infer underlying mechanisms that structure ecological systems and shape their resilience (Kéfi et al. 2007, Scanlon et al.

Manuscript received 8 December 2014; revised 2 April 2015; accepted 10 April 2015. Corresponding Editor: W. J. D. van Leeuwen.

${ }^{12}$ E-mail: xuchi@nju.edu.cn
2007). Although this is an exciting possibility, there are still major barriers, as similar patterns may emerge from different ecological processes (Levin 1992, McIntire and Fajardo 2009). So far, the clearest link between pattern and process has been found for regular patterns such as "tiger bush," where regular bands of vegetation interweaved with bare soil (e.g., in drylands) or sparser plant cover (e.g., in peatlands) resemble the stripes of a tiger skin (Macfadyen 1950, Foster et al. 1983). These regular patterns have been explained by the interplay between short-range positive feedbacks and long-range negative feedbacks on plant biomass (Tongway et al. 2001, Rietkerk and Van de Koppel 2008).

However, vegetation is more commonly characterized by irregular patchy structures rather than periodic 
spatial patterns. This is particularly true in drylands, where patchy vegetation patterns have been intensively studied (e.g., Macfadyen 1950, Tongway et al. 2001). An obvious mechanism producing plant spatial clustering is spatial variation in resources (e.g., nutrient or water availability) or in disturbance effects (e.g., herbivore or fire). Yet, patchy vegetation is also found in many seemingly homogeneous landscapes with no obvious disturbance effects. An alternative explanation for patch formation is vegetative expansion through roots and tillers; however one often finds individuals from different species clumping together (e.g., Fuentes et al. 1984). Facilitative interactions between neighboring plants are a prime potential explanation for clumped vegetation patterns in many harsh environments such as drylands, alpine systems, salt marshes, and boreal peat bogs. These facilitative interactions occur when there are overruling benefits in terms of growth or survival for plants growing in the proximity of nurse plants that can ameliorate abiotic conditions, improve resource acquisition, or reduce herbivore damage (Holmgren et al. 1997, Callaway 2007). The effects of these facilitative interactions on the composition and structure of plant communities often cascade across trophic levels affecting species diversity (Bruno et al. 2003), and ecosystem functioning and resilience to environmental changes $(\mathrm{Xu}$ et al. 2015). Inferring facilitation from spatial patterns, therefore, can play a pivotal role in linking ecosystem structure and function, to advance our understanding of how ecosystems may respond to environmental changes.

To estimate the frequency of facilitative interactions, ecologists combine labor-intensive field measurements of plant co-occurrences with field experiments that assess the mechanisms behind the correlations observed in the field. Studies relating facilitation with spatial association between plants in the field (e.g., Fuentes et al. 1984) or in combination with remotely sensed images (FuentesCastillo et al. 2012) have remained largely restricted to relatively small scales and accessible sites (but see Callaway et al. 2002, Cavieres et al. 2014, Soliveres et al. 2014). These limitations hinder our ability to compare the role of facilitation across large environmental gradients and biomes.

Here we evaluate whether remotely sensed spatial vegetation patterns can provide useful information to infer the frequency of plant positive co-occurrence on the ground, thus reflecting the potential importance of facilitation in arid plant communities. We used highresolution images from 65 drylands distributed across Africa, Australia, the Mediterranean Basin, South America, and North America, and related the spatial patterns to field assessments of plant positive cooccurrence as a proxy for facilitation.

\section{Materials And Methods}

Study sites and image analysis

We selected 65 sites from the data set of 224 global drylands compiled by Maestre et al. (2012, Fig. 1,
Appendix A). These sites have remotely sensed images at very high resolutions $(\leq 0.5 \mathrm{~m})$, available through Google Earth. For each study site, we took one snapshot from the most recent Google Earth image. Each acquired image was geometrically corrected with an accuracy of $<0.5$ pixel of root mean square error. An area of $250 \times 250 \mathrm{~m}$ without obvious human disturbances (e.g., artificial surfaces and watering points for livestock) was selected from each previously georeferenced image. We used standard supervised image classification techniques with the maximum likelihood method (Lillesand et al. 2004) to identify vegetation patches. Two classes (vegetated and non-vegetated) were generated using a supervised classification, and the achieved accuracy was over $90 \%$ for each study site (Appendices A and B). We focused on vegetation patches that can be clearly identified from Google Earth images. These include woody plants (trees and shrubs) and large tussock grasses, which represent the most common growth forms available in this global data set.

The most direct approach to study spatial association, such as point pattern analysis, is to analyze the distances between individual plants (Wiegand and Moloney 2004). From the remotely sensed images provided by Google Earth, this is only feasible in situations where we can distinguish each individual plant and if the occurrence of solitary plants is confirmed by field observations. We can then statistically determine whether the spatial distribution is significantly over-dispersed (clumpy) or under-dispersed (more regular than expected by chance; Appendix C). However, vegetation patches formed by multiple plants were common in most of the drylands studied. These composite patches make individual plants indistinguishable from the remotely sensed images, hampering the application of point pattern analysis. Therefore, we focused on patch-size distribution, which has been repeatedly shown to be an important attribute linked to ecological processes such as environmental stress and biotic interactions in drylands (Kéfi et al. 2007, Scanlon et al. 2007). We plotted the noncumulative patch-size distribution (i.e., the number of patches at a series of patch size classes) at each site using the binning method, which is a robust approach when distribution parameters do not need to be fit (White et al. 2008). The data were processed using ENVI 4.8 (Exelis, McLean, Virginia, USA), ArcGIS 10.0 (ESRI, Redlands, California, USA) and MATLAB R2011b (MathWorks, Natick, Massachusetts, USA).

\section{Patch-size distributions: remotely sensed vs. modelling predictions}

We used a very simple individual-based model to generate patch-size distributions using different assumptions about the type and strength of the plant-plant interactions, and compared these generated patch-size distributions with those obtained from the remotely sensed images. The purpose of this model is to find a minimal set of assumptions necessary to describe the

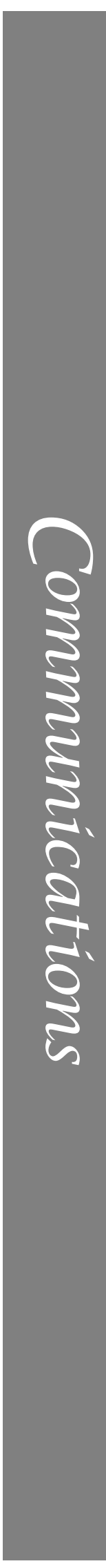



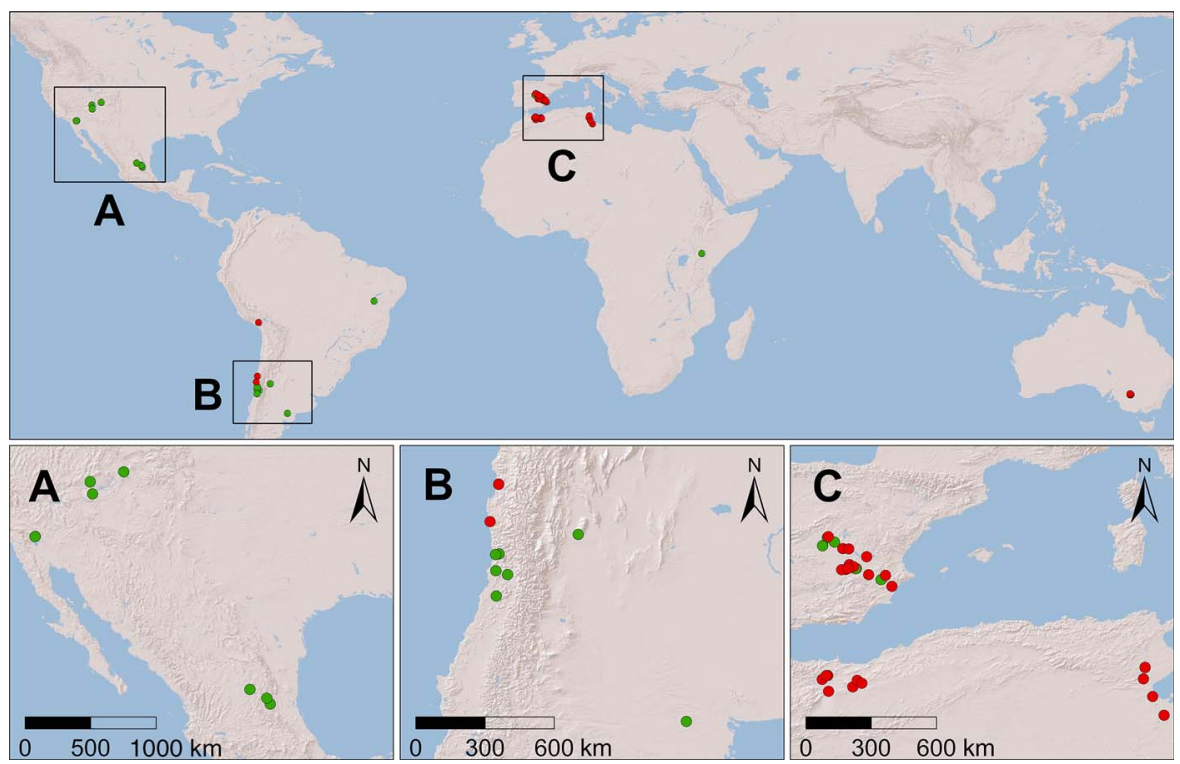

FIG. 1. Distribution of the 65 study sites selected from the global dryland database by Maestre et al. (2012). Red dots indicate the sites where plant co-occurrence was measured in situ, green dots show the sites without field measurement of plant co-occurrence.

patch-size distributions in the field. The model starts by randomly allocating individual plant sizes from a normal distribution. These plants are initially spread on a two-dimensional surface by drawing circles of normally distributed sizes at random positions; subsequently, some of these imaginary plants are eliminated to represent mortality. In the null model, mortality is independent of the distance to other plants (CSR, complete spatial randomness), but only depends on the harshness of the environment. We use the Pearson's moment coefficient of skewness as a metric to compare this null model with versions of the model where facilitation and competition are modelled as a mortality that depends on the distance to a neighboring plant. We assume that mortality due to competition decreases away from other plants. To mimic facilitation, we increase survival rates if other plants are nearby. Sigmoidal functions were used to model these relationships between mortality and distance. We then analyzed the resulting patch-size distributions, where patches are obtained by fusing circles that touch or overlap into a single patch (see Appendices D and E for model descriptions and results).

\section{Field assessment of plant positive co-occurrence}

We sampled plant co-occurrence in the field in 37 of the 65 study sites (Fig. 1, Appendix A). We selected potential nurse plants among the most abundant growth forms (i.e., trees, shrubs, and or large tussock grasses, depending on the dominant growth forms present within each site $)$ and sampled $30\left(0.5 \times 0.5 \mathrm{~m}^{2}\right)$ quadrats beneath these dominant microsites and in open areas between June 2009 and December 2011. Quadrats were chosen to include at least three trees, five shrubs, or 10 grasses and were at least $1 \mathrm{~m}$ apart. Quadrats sampled in open areas were located at least $2 \mathrm{~m}$ away from any of the potential nurse plants. Within each quadrat, we counted the number of individuals of each perennial species. Recording the abundance of perennial species is less sensitive to seasonal and climatic changes; thus, this abundance is a more reliable indicator of plant-plant interactions at the community level, and more suitable for comparisons across sites. Although annual plants may also be facilitated (Holzapfel et al. 2006), we did not include them in our sampling as they grow only during wet periods and survive dry seasons as seeds (Gutiérrez and Meserve 2003, Caballero et al. 2008).

Using these data, we assessed the degree of cooccurrence of each neighboring species with each nurse by comparing the number of individuals of the neighboring species growing beneath the nurse vs. those growing in the open areas (sampling effort was identical for both microsites, i.e., 30 quadrats). The significance of each pairwise co-occurrence was estimated by using the $\chi^{2}$ statistic. Neighboring species with more individuals growing beneath the nurse than expected by chance thus represent significant positive co-occurrence, which may reflect facilitative interaction between them. The frequency of positive associations within each site was measured as the percentage of the total pairwise target woody-nurse interactions observed in the community (see Soliveres et al. [2014] for more details).

\section{Results AND Discussion}

In the drylands studied, the frequency distribution of patch sizes was typically hump-shaped on a log scale, with a dominant mode representing abundant small patches and a distinct right tail representing fewer large 

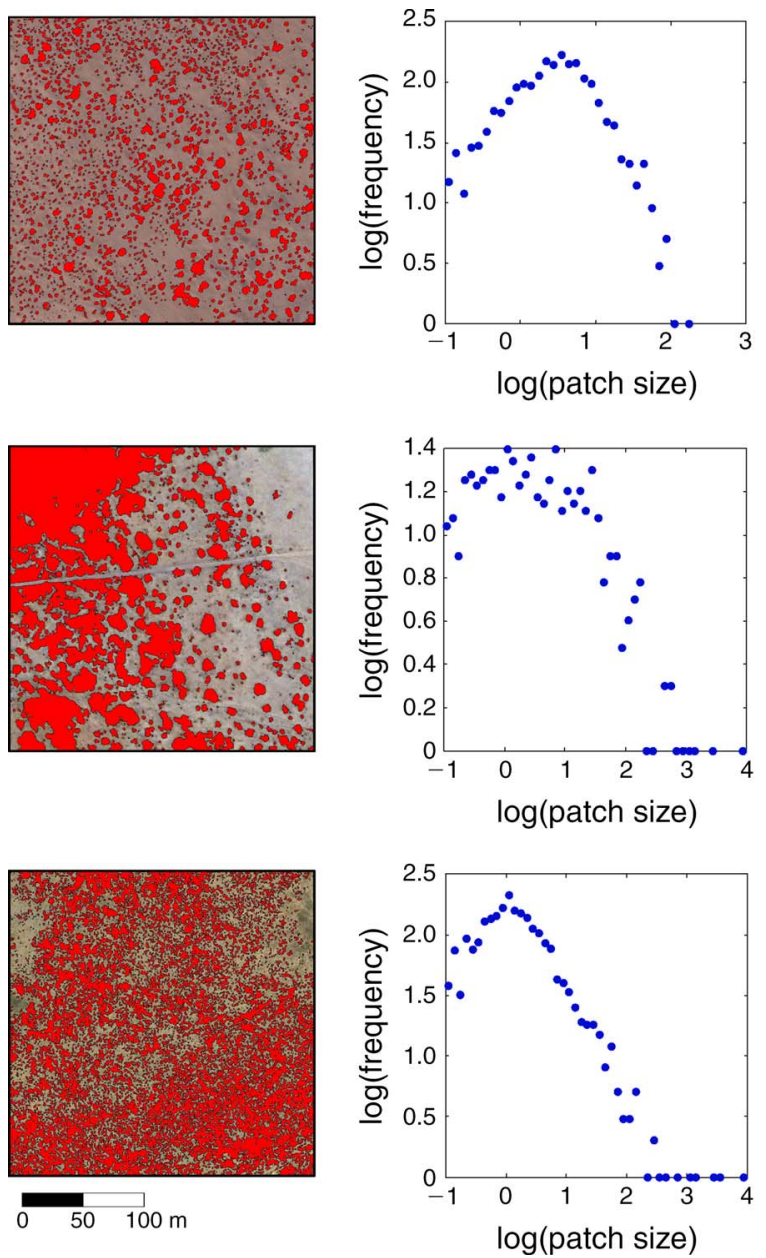
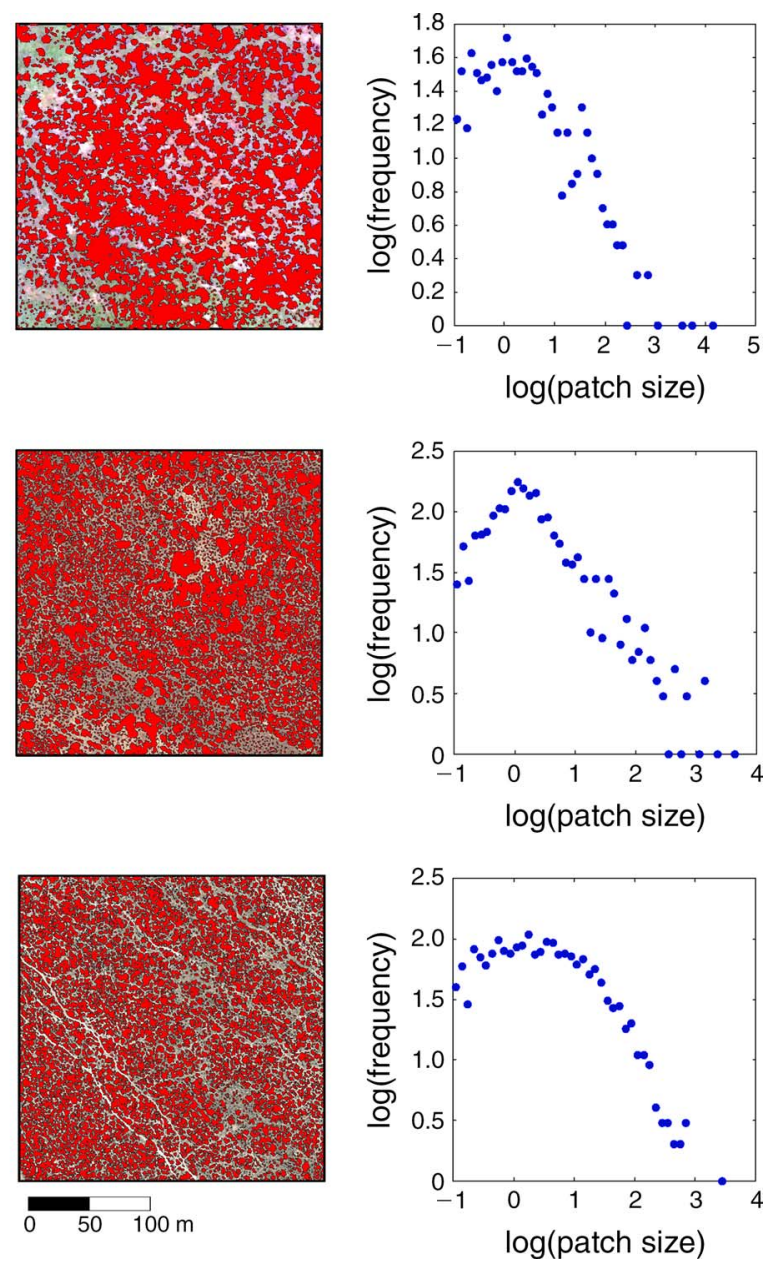

FIG. 2. Examples of vegetation patterns in the study sites with their corresponding patch-size distributions. The vegetation patches (red color) were extracted from the high-resolution Google Earth images. Patch size was measured in square meters.

patches (Fig. 2). An intuitive way to explain such a distribution would be that the mode roughly represents the typical size of single plants (or small patches), whereas the right tail is the result of large plant clusters. This would imply that the heaviness of the tail, reflected in the skewness of the distribution, could be a measure of the tendency for plants to grow together and form clusters. If these clusters were explained by facilitative interactions, the more skewed to the right the patch-size distribution is (i.e., toward bigger patches), the more frequent the positive association between plants is, and thus more likely facilitation would be acting.

Our model shows that facilitation typically produces a distinct right tail as a result of plant clusters that generate large patches. This results in a right-skewed patch-size distribution, as compared to the null model. By contrast, simulated competition results in distributions that are more left skewed than those from the null model (Fig. 3, Appendix E: Fig. E1). Skewness of the patch-size distribution is thus a simple and sensitive indicator of the way in which the shape of the patch-size distribution is affected by spatial association of individual plants as expected from the interplay of competition and facilitation. Obviously, plants will also be more likely to "touch" each other by chance and form clusters as total plant cover increases. This effect can be seen in the null model (entirely random spatial distribution, CSR), where skewness of the patch-size distribution increases almost linearly with total plant cover (Fig. 4, red line), until a cover of about $70 \%$. This is roughly the point where the so-called spanning cluster (Kéfi et al. 2011) arises that spreads across the entire area (the percolation point; Appendix E: Fig. E2).

Most of the patterns found in the remotely sensed images were more right skewed than the patterns from the null model simulating a random distribution. This suggests that the plants in the drylands studied show stronger spatial clustering than expected by chance, which is consistent with a scenario where increased survival of clusters of plants due to facilitation is the dominant interaction among plant species (circles in Fig. 4). To check this, we related the skewness results to our 
A) Facilitation
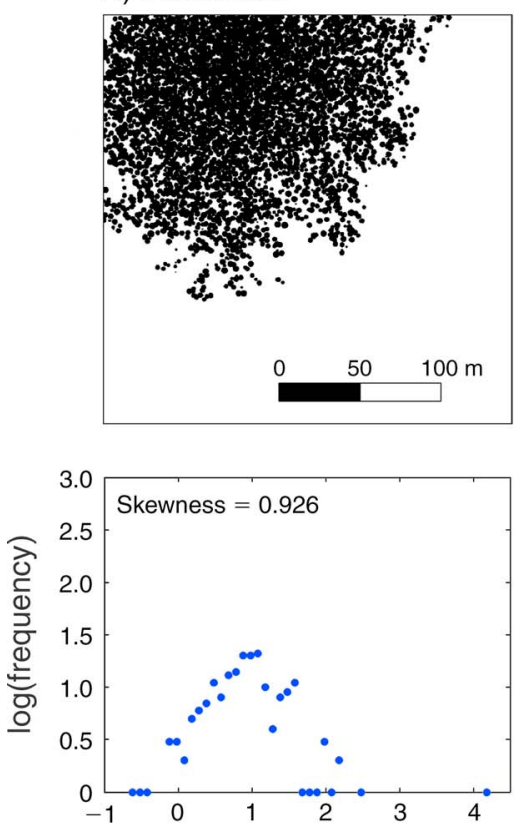

B) CSR
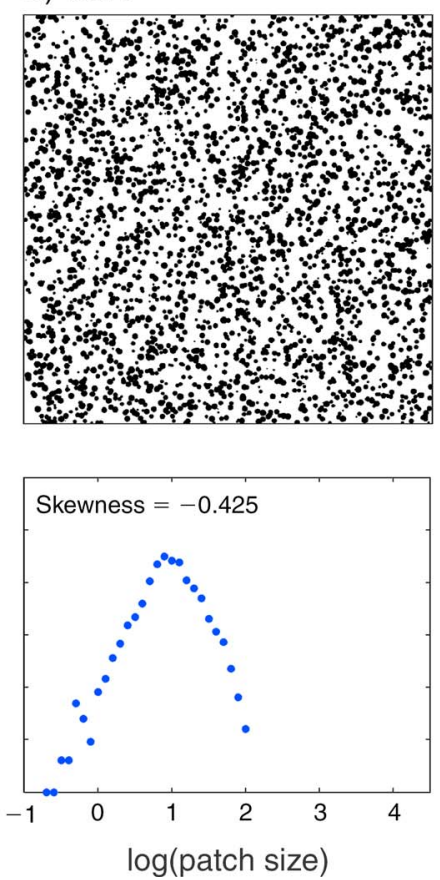

C) Competition
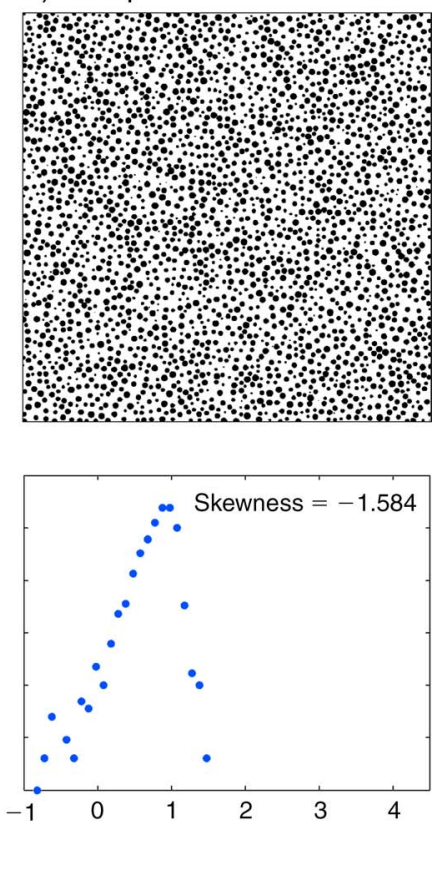

FIG. 3. Examples of spatial patterns (upper) and patch-size distribution (lower; Pearson's moment coefficient of skewness is shown in the lower panels) generated by the (A) facilitation, (B) null (complete spatial randomness, CSR), and (C) competition models at $30 \%$ plant cover. See model examples at different plant covers in Appendix E.

ground observations of plant positive co-occurrence. Indeed, the deviance of the remotely sensed patterns from the null model (calculated as skewness of patchsize distribution from the images subtracting that from the null model at corresponding plant cover values) was correlated to the indicator of plant positive co-occurrence measured on the ground (Fig. 5, Pearson's $r=$ $0.366, P=0.026$ ). This correlation was even stronger after correcting for the effect of plant cover based on partial correlation analysis (partial Pearson's $r=0.429$, $P=0.009$ ). We also assessed these correlations for the major vegetation types studied (i.e., grasslands, shrublands, and open woodlands) separately (Appendix F), and found significant linear relationships between the field measurement of plant co-occurrence and the remotely sensed skewness indicator both in woodlands ( $P=0.028, P=0.039$ after correcting for plant cover) and shrublands $(P=0.012, P<0.001$ after correcting for plant cover), but found weak correlations for grasslands $(P=0.824, P=0.557$ after correcting for plant cover). A meta-analysis of the published literature also found that grasses often have weaker facilitative effects than shrubs and trees (Gómez-Aparicio 2009).

The plant spatial clustering measured from remotely sensed images plausibly reflects facilitation, since such spatial pattern signal is strongly correlated with the plant positive co-occurrence we measured on the ground and it is also clearly indicated by our modelling results. Our results are consistent with the model predictions

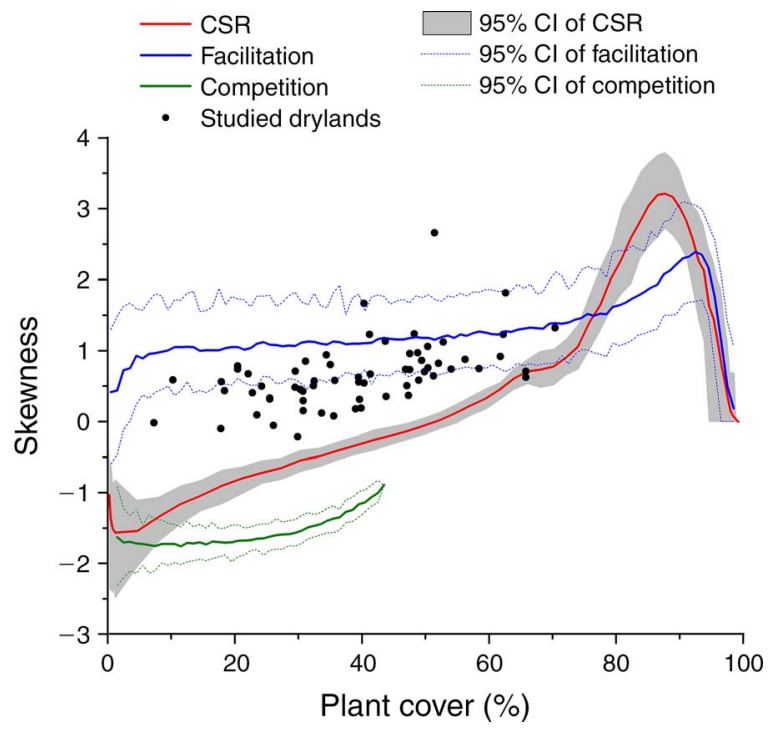

FIG. 4. Relationship between plant cover and the skewness (Pearson's moment coefficient) of patch-size distribution for the null model (complete spatial randomness, CSR), the facilitation model, the competition model and the remotely sensed data for the 65 drylands studied. In the competition model, the simulation is implemented only up to $<45 \%$ plant cover, as the repellent effect from existent individuals increasingly forbids the recruitment of more individuals. 

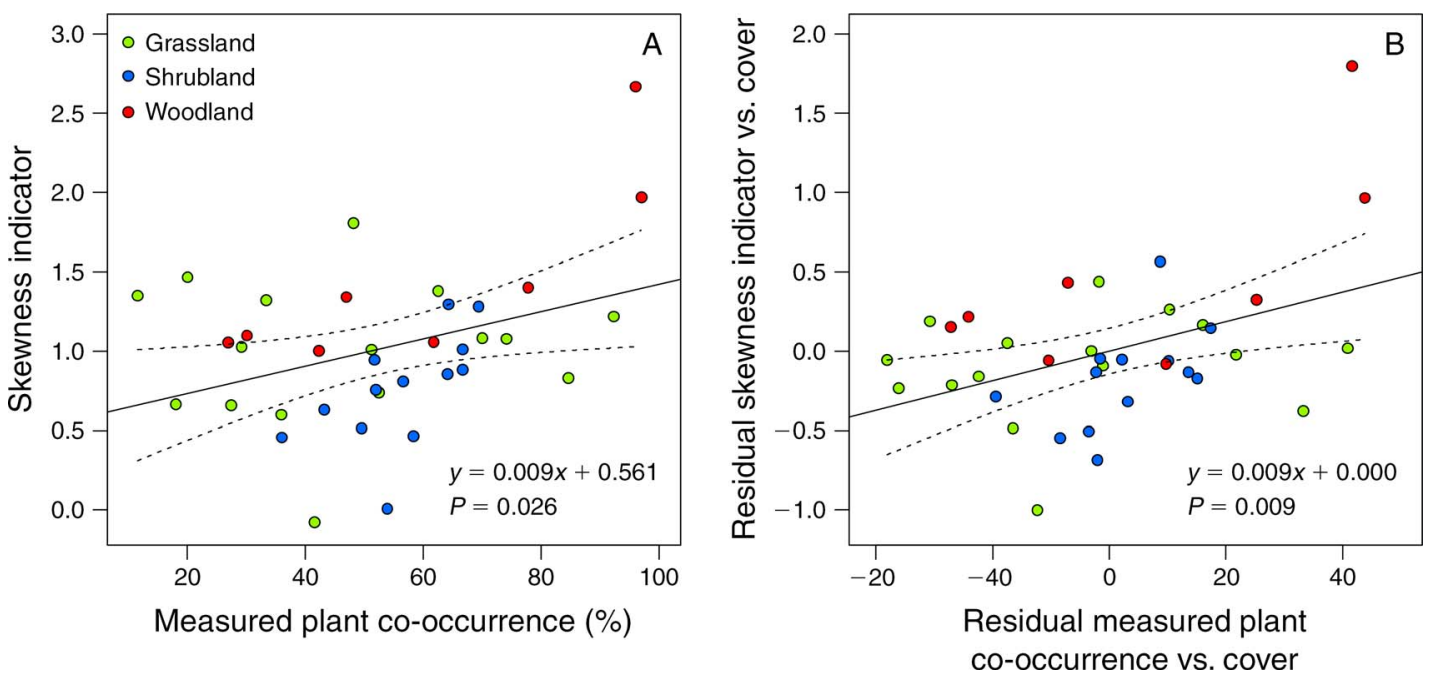

FIG. 5. Relationship between plant positive co-occurrence measured in situ and the Pearson's moment coefficient of skewness indicator derived from remote sensing and the null model (A) without correction and (B) with correction for plant cover using partial correlation analysis. The dashed lines represent $95 \%$ confidence intervals.

based on facilitative interactions. However, observational approaches and correlational evidence cannot rule out alternative explanations such as the role of underlying environmental gradients or dispersal limitation. Yet positive co-occurrences in drylands have been tightly linked to facilitative interactions demonstrated experimentally (e.g., Tirado and Pugnaire 2005) and commonly used as indicator of facilitation (e.g., Alados et al. 2006).

In the collection of sites across continents that we analyzed here, we did not observe Turing-like patterns (Deblauwe et al. 2012). While Turing patterns are easily recognizable, it may be that irregular patterns are in part dependent on the scale of study and on the plant groups addressed. Earlier work found power-law or truncated power-law patch-size distributions in dryland vegetation (Kéfi et al. 2007, Maestre and Escudero 2009). These distributions have been interpreted as a result of local facilitation generating more large patches than expected by chance (Kéfi et al. 2011). In contrast to the power-law patch-size distribution, we mostly found a lower frequency of small patches forming hump-shaped distributions, which is consistent with the idea that the observed patches at this scale reflect a combination of solitary plants (with a given size distribution) and multiplant patches (as a result of plant facilitation). A plausible explanation of such difference is that Kéfi et al. (2007) included small patches of grasses in their analyses, which could not be detected by the remotely sensed data.

\section{CONCLUSIONS AND IMPLICATIONS}

Our field ground validation indeed suggests that the features of the plant patch-size distribution retrieved from remotely sensed images can be used to infer the frequency of plant positive co-occurrences measured on the ground. Our results suggest a way to systematically map indicators of plant co-occurrence globally. This opens new opportunities for assessing the role of facilitation, and the effects of environmental conditions, such as ongoing climate change, on the balance between facilitation and competition in plant communities. Furthermore, by linking our approach to data on ecosystem functioning that can be obtained from remote sensing at global scales or by repeating our analyses across several years, we may substantially advance our knowledge on the role of plant interactions for maintaining ecosystem functioning. These new tools will never be able to replace the strength of field experimentation, but can help us to expand the scale of our enterprises and to identify places worth of examining through detailed experiments.

\section{ACKNOWLEDGMENTS}

We thank Eliot McIntire and two anonymous reviewers for their constructive comments on an earlier version of this work, and all the members of the EPES-BIOCOM network for the collection of field data. This work was partly funded by the European Research Council (ERC) Advanced Grant (268732) and the Spinoza Award to M. Scheffer. C. Xu was supported by National Natural Science Foundation of China (41271197). Field data collection was supported by the European Research Council under the European Community's Seventh Framework Programme (FP7/2007-2013)/ERC Grant (242658). P. A. Marquet and S. Abades were supported by Grants ICM-MINECON, P05-002 IEB and CONICYT PFB-0023. This is ISEM publication number ISEM 2015-090.

\section{Literature Cited}

Alados, C. L., P. Gotor, P. Ballester, D. Navas, J. M. Escos, T. Navarro, and B. Cabezudo. 2006. Association between competition and facilitation processes and vegetation spatial patterns in alpha steppes. Biological Journal of the Linnean Society $87: 103-113$. 
Bruno, J. F., J. J. Stachowicz, and M. D. Bertness. 2003 Inclusion of facilitation into ecological theory. Trends In Ecology \& Evolution 18:119-125.

Caballero, I., J. M. Olano, A. Escudero, and J. Loidi. 2008. Seed bank spatial structure in semi-arid environments: beyond the patch-bare area dichotomy. Plant Ecology 195:215-223.

Callaway, R. M. 2007. Positive interactions and interdependence in plant communities. Springer, Dordrecht, The Netherlands.

Callaway, R. M., R. Brooker, P. Choler, Z. Kikvidze, C. J. Lortie, R. Michalet, L. Paolini, F. I. Pugnaire, B. Newingham, and E. T. Aschehoug. 2002. Positive interactions among alpine plants increase with stress. Nature 417:844848.

Cavieres, L. A., R. W. Brooker, B. J. Butterfield, B. J. Cook, Z. Kikvidze, C. J. Lortie, R. Michalet, F. I. Pugnaire, C. Schöb, and S. Xiao. 2014. Facilitative plant interactions and climate simultaneously drive alpine plant diversity. Ecology Letters 17:193-202.

Cramer, M. D., and N. N. Barger. 2013. Are Namibian "fairy circles" the consequence of self-organizing spatial vegetation patterning? PLoS ONE 8:e70876.

Deblauwe, V., P. Couteron, J. Bogaert, and N. Barbier. 2012. Determinants and dynamics of banded vegetation pattern migration in arid climates. Ecological Monographs 82:3-21.

Foster, D., G. King, P. Glaser, and H. Wright. 1983. Origin of string patterns in boreal peatlands. Nature 306:256-258.

Fuentes, E. R., R. D. Otaiza, M. C. Alliende, A. Hoffmann, and A. Poiani. 1984. Shrub clumps of the Chilean matorral vegetation: structure and possible maintenance mechanisms. Oecologia 62:405-411.

Fuentes-Castillo, T., A. Miranda, A. Rivera-Hutinel, C. SmithRamírez, and M. Holmgren. 2012. Nucleated regeneration of semiarid sclerophyllous forests close to remnant vegetation. Forest Ecology and Management 274:38-47.

Gómez-Aparicio, L. 2009. The role of plant interactions in the restoration of degraded ecosystems: a meta-analysis across life-forms and ecosystems. Journal of Ecology 97:1202-1214.

Gutiérrez, J. R., and P. L. Meserve. 2003. El Niño effects on soil seed bank dynamics in north-central Chile. Oecologia 134:511-517.

Holmgren, M., M. Scheffer, and M. A. Huston. 1997. The interplay of facilitation and competition in plant communities. Ecology 78:1966-1975.

Holzapfel, C., K. Tielbörger, H. A. Parag, J. Kigel, and M. Sternberg. 2006. Annual plant-shrub interactions along an aridity gradient. Basic and Applied Ecology 7:268-279.

Kéfi, S., M. Rietkerk, C. L. Alados, Y. Pueyo, V. P. Papanastasis, A. ElAich, and P. C. de Ruiter. 2007. Spatial vegetation patterns and imminent desertification in mediterranean arid ecosystems. Nature 449:213-217.
Kéfi, S., M. Rietkerk, M. Roy, A. Franc, P. C. de Ruiter, and M. Pascual. 2011. Robust scaling in ecosystems and the meltdown of patch size distributions before extinction. Ecology Letters 14:29-35.

Levin, S. A. 1992. The problem of pattern and scale in ecology. Ecology 73:1943-1967.

Lillesand, T. M., R. W. Kiefer, and J. W. Chipman. 2004. Remote sensing and image interpretation. John Wiley, Hoboken, New Jersey, USA.

Macfadyen, W. 1950. Vegetation patterns in the semi-desert plains of British Somaliland. Geographical Journal 116:199211.

Maestre, F. T., and A. Escudero. 2009. Is the patch size distribution of vegetation a suitable indicator of desertification processes? Ecology 90:1729-1735.

Maestre, F. T., et al. 2012. Plant species richness and ecosystem multifunctionality in global drylands. Science 335:214-218.

McIntire, E. J., and A. Fajardo. 2009. Beyond description: the active and effective way to infer processes from spatial patterns. Ecology 90:46-56.

Rietkerk, M., and J. Van de Koppel. 2008. Regular pattern formation in real ecosystems. Trends in Ecology \& Evolution 23:169-175.

Scanlon, T. M., K. K. Caylor, S. A. Levin, and I. RodriguezIturbe. 2007. Positive feedbacks promote power-law clustering of Kalahari vegetation. Nature 449:209-212.

Soliveres, S., et al. 2014. Functional traits determine plant cooccurrence more than environment or evolutionary relatedness in global drylands. Perspectives in Plant Ecology, Evolution and Systematics 16:164-173.

Thompson, S., and G. Katul. 2011. Inferring ecosystem parameters from observation of vegetation patterns. Geophysical Research Letters 38:L20401.

Tirado, R., and F. I. Pugnaire. 2005. Community structure and positive interactions in constraining environments. Oikos 111:437-444

Tongway, D. D. J., C. Valentin, and J. Seghieri. 2001. Banded vegetation patterning in arid and semi-arid environments: ecological processes and consequences for management. Springer, New York, New York, USA.

White, E. P., B. J. Enquist, and J. L. Green. 2008. On estimating the exponent of power-law frequency distributions. Ecology 89:905-912.

Wiegand, T., and K. A. Moloney. 2004. Rings, circles, and nullmodels for point pattern analysis in ecology. Oikos 104:209229.

Xu, C., E. H. Van Nes, M. Holmgren, S. Kéfi, and M. Scheffer. 2015. Local facilitation may cause tipping points on a landscape level preceded by early warning indicators. American Naturalist. http://dx.doi.org/10.1086/682674

\section{Supplemental Material}

Ecological Archives

Appendices A-F are available online: http://dx.doi.org/10.1890/14-2358.1.sm 